\title{
A lower bound for the Graver complexity of the incidence matrix of a complete bipartite graph
}

\author{
Taisei Kudo and Akimichi Takemura*
}

\begin{abstract}
We give an exponential lower bound for the Graver complexity of the incidence matrix of a complete bipartite graph of arbitrary size. Our result is a generalization of the result by Berstein and Onn [2] for the complete bipartite graph $K_{3, r}, r \geq 3$.
\end{abstract}

KEYWORdS AND PHRASES: Algebraic statistics, contingency table, threeway transportation program.

\section{Introduction and the main result}

The Graver complexity of an integer matrix is currently actively investigated for its importance to integer programming, algebraic statistics and other applications $[2,4,5,1]$. In particular, from the universality of the three-way transportation program to general integer programs $[3,6]$, the Graver complexity of the incidence matrix of the complete bipartite graph $K_{3, r}$ is particularly important. Berstein and Onn [2] proved that the Graver complexity $g(r)$ for the incidence matrix of $K_{3, r}, r \geq 3$ is bounded below as $g(r)=\Omega\left(2^{r}\right)$, where $g(r) \geq 17 \cdot 2^{r-3}-7$. It is a natural question to generalize this result to the complete bipartite graph $K_{t, r}$ of arbitrary size $t, r$. We prove that the Graver complexity for $K_{t, r}$ is $\Omega\left((t-1)^{r}\right)$, where $t \geq 4$ is fixed and $r$ diverges to infinity. For proving our result, we employ double induction on $r$ and $t$ starting from the result of [2].

Let $A_{t, r}$ denote the incidence matrix of the complete bipartite graph $K_{t, r}$ and let $g\left(A_{t, r}\right)$ denote its Graver complexity. Here we state our main theorem. Relevant notations and definitions will be given in the next section.

Theorem 1.1. The Graver complexity of $A_{t, r}$ for any $4 \leq t \leq r$ is bounded from below as

$$
g\left(A_{t, r}\right) \geq(t-1)^{r-t}\left(b_{t}+\frac{1}{t-2}\right)-\frac{1}{t-2},
$$

arXiv: 1102.4674

*JST, CREST. 
where

$$
b_{t}=(t-2) !\left(15+\sum_{i=1}^{t-4} \frac{i+4}{(i+2) !}\right) .
$$

We give a proof of this theorem in Section 3 after giving necessary definitions and reviewing relevant known results in Section 2. We conclude the paper with some discussion in Section 4.

\section{Preliminaries}

In this section, we summarize our notation and review relevant known results on the Graver complexity following [2].

The integer kernel of an $s \times t$ integer matrix $A$ is denoted by $\operatorname{ker}_{\mathbb{Z}}(A)=$ $\left\{x \in \mathbb{Z}^{t} \mid A x=0\right\}$. Define a partial order $\sqsubseteq$ on $\mathbb{Z}^{t}$, which extends the coordinate-wise order $\leq$ on $\mathbb{Z}_{+}^{t}$, as follows: For two vectors $u, v \in \mathbb{Z}^{t}, u \sqsubseteq v$ if $\left|u_{i}\right| \leq\left|v_{i}\right|$ and $u_{i} v_{i} \geq 0$ for $i=1, \ldots, t$. The Graver basis $\mathcal{G}(A)$ of $A$ is the finite set of $\sqsubseteq$-minimal elements in the set $\operatorname{ker}_{\mathbb{Z}}(A) \backslash\{0\}$.

For any fixed positive integer $h$, write an $h t$-dimensional integer vector $x \in \mathbb{Z}^{h t}$ as $x=\left(x^{1}, \ldots, x^{h}\right)$ with each block $x^{i}$ belonging to $\mathbb{Z}^{t}$. The type of $x=\left(x^{1}, \ldots, x^{h}\right)$ is the number type $(x):=\#\left(\left\{i \mid x^{i} \neq 0\right\}\right)$ of nonzero blocks of $x$. The $h$-th Lawrence lifting of an $s \times t$ matrix $A$ is the following $(t+h s) \times h t$ matrix, with $I_{t}$ denoting the $t \times t$ identity matrix:

$$
A^{(h)}:=\left(\begin{array}{ccccc}
A & 0 & 0 & \ldots & 0 \\
0 & A & 0 & \ldots & 0 \\
\vdots & \vdots & \ddots & \vdots & \vdots \\
0 & 0 & 0 & \ldots & A \\
I_{t} & I_{t} & I_{t} & \ldots & I_{t}
\end{array}\right) .
$$

The Graver complexity of $A$ is defined as

$$
g(A)=\sup \left(\{0\} \cup\left\{\operatorname{type}(x) \mid x \in \bigcup_{h \geq 1} \mathcal{G}\left(A^{(h)}\right)\right\}\right) .
$$

Let $\mathcal{G}(\mathcal{G}(A))$ denote the Graver basis of a matrix whose columns are the elements of $\mathcal{G}(A)$ ordered arbitrarily. The following result shows that the Graver complexity of $A$ is determined by $\mathcal{G}(\mathcal{G}(A))$.

Proposition 2.1. [7] The Graver complexity of A satisfies

$$
g(A)=\max \left\{\|x\|_{1}: x \in \mathcal{G}(\mathcal{G}(A))\right\},
$$

where $\|\cdot\|_{1}$ denotes the 1-norm of a vector. 
A circuit of an integer matrix $A$ is a nonzero integer vector $x \in \operatorname{ker}_{\mathbb{Z}}(A)$, that has inclusion-minimal support with respect to $\operatorname{ker}_{\mathbb{Z}}(A)$ and whose nonzero entries are relatively prime. Let $\mathcal{C}(A)$ denote the set of circuits of a matrix $A$. Then $\mathcal{C}(A) \subseteq \mathcal{G}(A)$ (cf. [8]). An integer relation $h=\left(h_{1}, \ldots, h_{k}\right)$ on integer vectors $v^{1}, \ldots, v^{k} \in \mathbb{Z}^{t}$

$$
0=h_{1} v^{1}+\cdots+h_{k} v^{k}
$$

is primitive if $h_{1}, \ldots, h_{k}$ are relatively prime positive integers and no $k-1$ of the $\left\{v^{i}\right\}_{i=1}^{k}$ satisfy any nontrivial linear relation. By $\mathcal{C}(A) \subseteq \mathcal{G}(A)$ and Proposition 2.1, we have the following result.

Proposition 2.2. [2] Suppose that $h$ is a primitive relation on some set of circuits $\left\{x^{i}\right\}_{i=1}^{k}$ of an integer matrix A. Then the Graver complexity of $A$ satisfies $g(A) \geq \sum_{i=1}^{k} h_{i}$.

In this paper, we consider the Graver complexity of the incidence matrix $A_{t, r}$ for the complete bipartite graph $K_{t, r}$. Let $\mathbf{1}_{t}=(1,1, \ldots, 1)$ denote the $1 \times t$ matrix consisting of 1 's. Then the $r$-th Lawrence lifting $A_{t, r}=\mathbf{1}_{t}^{(r)}$ of $\mathbf{1}_{t}$ is the incidence matrix of $K_{t, r}$. In algebraic statistics, $A_{t, r}$ is the design matrix specifying the row sums and the column sums of a two-way contingency table. Another Lawrence lifting $\left(\mathbf{1}_{t}^{(r)}\right)^{(h)}$ of $\mathbf{1}_{t}^{(r)}$ is the design matrix for no-three-factor interaction model for $t \times r \times h$ three-way contingency tables $[1,5]$. It is also the coefficient matrix for the three-way transportation program. The Graver complexity $g\left(A_{t, r}\right)=g\left(\mathbf{1}_{t}^{(r)}\right)$ gives the bound of complexity of the Graver basis for the toric ideal associated with the nothree-factor interaction model for $t \times r \times h$ three-way contingency tables as $h \rightarrow \infty$.

We employ below the following notation, where $t, r$ are positive integers. Let

$$
V:=\left\{v_{1}, \ldots, v_{t}\right\}, U:=\left\{u_{1}, \ldots, u_{r}\right\} .
$$

Then $V \oplus U$ and $V \times U$ denote the set of vertices and the set of edges of the complete bipartite graph $K_{t, r}$, respectively. They index the rows and the columns of the incidence matrix $A_{t, r}$ of $K_{t, r}$. Here we explain interpretations of a circuit of $A_{t, r}$ referring to [2]. We interpret each vector $x \in \mathbb{Z}^{V \times U}$ as:

1. an integer valued function on the set of edges $V \times U$;

2. a $t \times r$ matrix with its rows and columns indexed by $\mathrm{V}$ and $\mathrm{U}$. 
Table 1: Circuits for $A_{3,4}$ in a $3 \times 4$ matrix form

\begin{tabular}{|c|c|c|c|c|c|}
\hline & $u_{1}$ & $u_{2}$ & $u_{3}$ & $u_{4}$ & \\
\hline \multirow{3}{*}{$x^{1}=\left(v_{1}, u_{4}, v_{3}, u_{2}, v_{2}, u_{3}\right)=$} & 0 & 0 & -1 & 1 & $v_{1}$ \\
\hline & 0 & -1 & 1 & 0 & $v_{2}$ \\
\hline & 0 & 1 & 0 & -1 & $v_{3}$ \\
\hline \multirow{3}{*}{$x^{2}=\left(v_{1}, u_{2}, v_{3}, u_{3}, v_{2}, u_{1}\right)=$} & -1 & 1 & 0 & 0 & $v_{1}$ \\
\hline & 1 & 0 & -1 & 0 & $v_{2}$ \\
\hline & 0 & -1 & 1 & 0 & $v_{3}$ \\
\hline \multirow{3}{*}{$x^{3}=\left(v_{1}, u_{4}, v_{2}, u_{1}, v_{3}, u_{2}\right)=$} & 0 & -1 & 0 & 1 & $v_{1}$ \\
\hline & 1 & 0 & 0 & -1 & $v_{2}$ \\
\hline & -1 & 1 & 0 & 0 & $v_{3}$ \\
\hline \multirow{3}{*}{$x^{4}=\left(v_{1}, u_{4}, v_{2}, u_{2}, v_{3}, u_{1}\right)=$} & -1 & 0 & 0 & 1 & $v_{1}$ \\
\hline & 0 & 1 & 0 & -1 & $v_{2}$ \\
\hline & 1 & -1 & 0 & 0 & $v_{3}$ \\
\hline \multirow{3}{*}{$x^{5}=\left(v_{1}, u_{1}, v_{2}, u_{2}, v_{3}, u_{3}\right)=$} & 1 & 0 & -1 & 0 & $v_{1}$ \\
\hline & -1 & 1 & 0 & 0 & $v_{2}$ \\
\hline & 0 & -1 & 1 & 0 & $v_{3}$ \\
\hline \multirow{3}{*}{$x^{6}=\left(v_{1}, u_{3}, v_{2}, u_{4}, v_{3}, u_{2}\right)=$} & 0 & -1 & 1 & 0 & $v_{1}$ \\
\hline & 0 & 0 & -1 & 1 & $v_{2}$ \\
\hline & 0 & 1 & 0 & -1 & $v_{3}$ \\
\hline \multirow{3}{*}{$x^{7}=\left(v_{1}, u_{2}, v_{2}, u_{3}, v_{3}, u_{4}\right)=$} & 0 & 1 & 0 & -1 & $v_{1}$ \\
\hline & 0 & -1 & 1 & 0 & $v_{2}$ \\
\hline & 0 & 0 & -1 & 1 & $v_{3}$ \\
\hline
\end{tabular}

With these interpretations, $x$ is in $\mathcal{C}\left(A_{t, r}\right)$ if and only if:

1. as a function on $V \times U$ with the following properties: its support is a circuit of $K_{t, r}$, along which its values \pm 1 alternate. It can be expressed by the sequence $\left(v_{i_{1}}, u_{i_{1}}, v_{i_{2}}, u_{i_{2}}, \ldots, v_{i_{l}}, u_{i_{l}}\right)$ of vertices of the circuit of $K_{t, r}$ on which it is supported, with the convention that its value is +1 on the first edge $\left(v_{i_{1}}, u_{i_{1}}\right)$.

2. as a nonzero matrix with the following properties: its elements are $0, \pm 1$, its row sums and columns sums are zeros, and it has an inclusionminimal support with respect to these properties.

The following example is the base case for our inductive argument for the lower bound of the Graver complexity.

Example 2.1. [2] Let $t=3$ and $r=4$. Consider seven circuits $\left\{x^{i}\right\}_{i=1}^{7}$ in Table 1 (written in a $3 \times 4$ matrix form) of $A_{3,4}=(1,1,1)^{(4)}$. They satisfy a primitive relation

$$
x^{1}+2 x^{2}+3 x^{3}+3 x^{4}+5 x^{5}+6 x^{6}+7 x^{7}=0 .
$$


Therefore, from Proposition 2.2

$$
g\left(A_{3,4}\right) \geq 1+2+3+3+5+6+7=27 .
$$

\section{Proof of the main theorem}

In this section, we give a proof of our main theorem. Our proof is based on recursive construction of primitive relations for circuits of $A_{t, r}$. We need recursions for $t$ and $r$, separately. In Lemma 3.1, we give a recursion for $t$, and in Lemma 3.2 we give a recursion for $r$.

Lemma 3.1. Let $t \geq 4$. Suppose that there are circuits $\left\{x^{i}\right\}_{i=1}^{k}$ of $A_{t, t+1}=$ $\mathbf{1}_{t}^{(t+1)}$ admitting a primitive relation $h$, where the $k$-th circuit and the $k$-th coefficient are

$$
\begin{aligned}
& x^{k}=\left(v_{1}, u_{2}, v_{2}, u_{3}, \ldots, v_{t}, u_{t+1}\right), \\
& h_{k}=1 .
\end{aligned}
$$

Then there are circuits $\left\{\bar{x}^{i}\right\}_{i=1}^{k+t}$ of $A_{t+1, t+1}=\mathbf{1}_{t+1}^{(t+1)}$ admitting a primitive relation $\bar{h}$, where the $(k+t)$-th circuit and the $(k+t)$-th coefficient are

$$
\begin{aligned}
& \bar{x}^{k+t}=\left(v_{1}, u_{1}, v_{2}, u_{2}, \ldots, v_{t+1}, u_{t+1}\right), \\
& \bar{h}_{k+t}=1 .
\end{aligned}
$$

Proof. Using the natural embedding of $K_{t, t+1}$ into $K_{t+1, t+1}$, we can interpret circuits of the former also as circuits of the latter. Put

$$
y^{i}=x^{i}, \forall i=1, \ldots, k-1,
$$

and define

$$
\begin{aligned}
y^{k+j-1} & =\left(v_{1}, u_{j}, v_{t+1}, u_{j+1}\right), \forall j=1, \ldots, t, \\
y^{k+t} & =\left(v_{1}, u_{2}, v_{2}, u_{3}, \ldots, v_{t}, u_{t+1}, v_{t+1}, u_{1}\right) .
\end{aligned}
$$

Table 2 displays $\left\{y^{k+j-1}\right\}_{j=1}^{t+1}$ as matrices, where

$$
p=(1,0, \ldots, 0,-1)^{\top} \in \mathbb{Z}^{t+1} .
$$

Blank entries are zeros. Note that these circuits satisfy $\sum_{j=1}^{t+1} y^{k+j-1}=x^{k}$. 
Table 2: Circuits for recursion on $t$

\begin{tabular}{|c|c|c|c|c|c|c|}
\hline & $u_{1}$ & $u_{2}$ & $u_{3}$ & $\ldots$ & $u_{t}$ & $u_{t+1}$ \\
\hline$y^{k}=\left(v_{1}, u_{1}, v_{t+1}, u_{2}\right)=$ & $p$ & $-p$ & & & & \\
\hline$y^{k+1}=\left(v_{1}, u_{2}, v_{t+1}, u_{3}\right)=$ & & $p$ & $-p$ & & & \\
\hline$\vdots$ & & & $\ddots$ & $\ddots$ & & \\
\hline$y^{k+t-1}=\left(v_{1}, u_{t}, v_{t+1}, u_{t+1}\right)=$ & & & & $p$ & $-p$ & \\
\hline$y^{k+t-1}=\left(v_{1}, u_{t}, v_{t+1}, u_{t+1}\right)=$ & & & & & $p$ & $-p$ \\
\hline & -1 & $\begin{array}{c}1 \\
-1\end{array}$ & 1 & & & \\
\hline$y^{k+t}=\left(v_{1}, u_{2}, v_{2}, u_{3}, \ldots, v_{t}, u_{t+1}, v_{t+1}, u_{1}\right)=$ & 1 & & $\ddots$ & $\begin{array}{l}\ddots \\
-1\end{array}$ & $\begin{array}{c}1 \\
-1\end{array}$ & $\begin{array}{c}1 \\
-1\end{array}$ \\
\hline
\end{tabular}

Suppose that $\bar{h} \in \mathbb{Z}^{k+t}$ satisfies

$$
\begin{aligned}
\bar{h}_{i} & =h_{i}, \quad \forall i=1, \ldots, k-1, \\
\bar{h}_{k+j-1} & =h_{k}, \quad \forall j=1, \ldots, t+1 .
\end{aligned}
$$

Then

$$
\sum_{i=1}^{k+t} \bar{h}_{i} y^{i}=\sum_{i=1}^{k-1} h_{i} y^{i}+\sum_{j=1}^{t+1} h_{k} y^{k+j-1}=\sum_{i=1}^{k-1} h_{i} x^{i}+h_{k} x^{k}=0 .
$$

Therefore, $\bar{h}$ is an integer relation of circuits $\left\{y^{i}\right\}_{i=1}^{k+t}$.

Next, we show that $\bar{h}$ is primitive. Suppose that $h^{\prime} \in \mathbb{Z}^{k+t}$ is a nontrivial relation on the $\left\{y^{i}\right\}_{i=1}^{k+t}$. Without loss of generality, we may assume that the $\left\{h_{i}^{\prime}\right\}_{i=1}^{k+t}$ are relatively prime integers, at least one of which is positive. We look at the row of $v_{t+1}$. Then it follows that

$$
h_{k}^{\prime}=h_{k+1}^{\prime}=\cdots=h_{k+t}^{\prime} .
$$

Therefore,

$$
0=\sum_{i=1}^{k+t} h_{i}^{\prime} y^{i}=\sum_{i=1}^{k-1} h_{i}^{\prime} y^{i}+h_{k}^{\prime} \sum_{j=1}^{t+1} y^{k+j-1}=\sum_{i=1}^{k-1} h_{i}^{\prime} x^{i}+h_{k}^{\prime} x^{k} .
$$


This is an integer relation on $\left\{x^{i}\right\}_{i=1}^{k}$, and because $h$ is primitive,

$$
h_{i}^{\prime}=h_{i}, \forall i=1, \ldots, k .
$$

Therefore, $h^{\prime}=\bar{h}$ and $\bar{h}$ is primitive.

Now apply to $\left\{y^{i}\right\}_{i=1}^{k+t}$ a permutation of columns so that $y^{k+t}$ becomes $\left(v_{1}, u_{1}, v_{2}, u_{2}, \ldots, v_{t+1}, u_{t+1}\right)$. For $i=1, \ldots, k+t$, let $\bar{x}^{i}$ be the circuit of $A_{t+1, t+1}$ which is the image of $y^{i}$ under this permutation. Then $\left\{\bar{x}^{i}\right\}_{i=1}^{k+t}$ also satisfy the primitive relation $\sum_{i=1}^{k+t} \bar{h}_{i} \bar{x}^{i}=0$ with the same coefficients $\bar{h}$. This completes the proof.

Lemma 3.2. Let $r \geq t \geq 4$. Suppose that there are circuits $\left\{x^{i}\right\}_{i=1}^{k}$ of $A_{t, r}=\mathbf{1}_{t}^{(r)}$ admitting a primitive relation $h$, where the $k$-th circuit and the $k$-th coefficient are

$$
\begin{aligned}
& x^{k}=\left(v_{1}, u_{r-t+1}, v_{2}, u_{r-t+2}, \ldots, v_{t}, u_{r}\right), \\
& h_{k}=1 .
\end{aligned}
$$

Then there are circuits $\left\{\bar{x}^{i}\right\}_{i=1}^{k+t-1}$ of $A_{t, r+1}=\mathbf{1}_{t}^{(r+1)}$ admitting primitive relation $\bar{h}$, where the $(k+t-1)$-th circuit is

$$
\bar{x}^{k+t-1}=\left(v_{1}, u_{r-t+2}, v_{2}, u_{r-t+3}, \ldots, v_{t}, u_{r+1}\right)
$$

and the elements of $\bar{h}$ are

$$
\bar{h}_{i}=(t-1) h_{i}, \forall i=1, \ldots, k-1, \bar{h}_{k}=\bar{h}_{k+1}=\cdots=\bar{h}_{k+t-1}=h_{k}=1 .
$$

Proof. Using the natural embedding of $K_{t, r}$ into $K_{t, r+1}$, we can interpret circuits of the former also as circuits of the latter. Put

$$
y^{i}=x^{i}, \forall i=1, \ldots, k-1,
$$

and for all $j=1, \ldots, t$, let $y^{k+j-1}$ denote vectors obtained by changing vertex $u_{r-j+1}$ of $x^{k}$ to $u_{r+1}$. Table 3 displays these circuits as matrices. Here for each $i=1, \ldots, t, q^{i} \in \mathbb{Z}^{t}$ denotes a vector satisfying

$$
q_{i}^{i}=1, q_{i+1}^{i}=-1,
$$

and the rest are zeros. Here we identify $t+1$ with 1 .

Notice that

$$
\sum_{j=1}^{t} y^{k+j-1}=(t-1) x^{k} .
$$


Table 3: Circuits for recursion on $r$

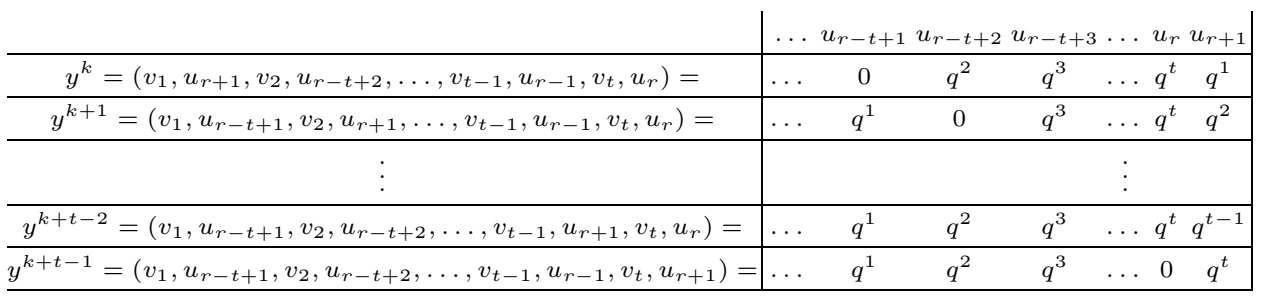

Define

$$
\begin{aligned}
\bar{h}_{i} & =(t-1) h_{i}, \forall i=1, \ldots, k-1, \\
\bar{h}_{k+j-1} & =h_{k}=1, \quad \forall j=1, \ldots, t+1 .
\end{aligned}
$$

Then

$$
\sum_{i=1}^{k+t-1} \bar{h}_{i} y^{i}=\sum_{i=1}^{k-1}(t-1) h_{i} y^{i}+\sum_{j=1}^{t} h_{k} y^{k+j-1}=\sum_{i=1}^{k-1} h_{i} x^{i}+h_{k} x^{k}=0 .
$$

Therefore, $\bar{h}$ is an integer relation on circuits $\left\{y^{i}\right\}_{i=1}^{k+t-1}$.

Next we show that $\bar{h}$ is primitive. Suppose that $h^{\prime} \in \mathbb{Z}^{k+t-1}$ is a nontrivial relation on the $\left\{y^{i}\right\}_{i=1}^{k+t-1}$. Without loss of generality, we may assume that $\left\{h_{i}^{\prime}\right\}_{i=1}^{k+t-1}$ are relatively prime integers, at least one of which is positive. Consider the column of $u_{r+1}$. Then

$$
h_{k}^{\prime}=h_{k+1}^{\prime}=\cdots=h_{k+t-1}^{\prime} .
$$

Therefore,

$$
0=\sum_{i=1}^{k+t-1} h_{i}^{\prime} y^{i}=\sum_{i=1}^{k-1} h_{i}^{\prime} y^{i}+h_{k}^{\prime} \sum_{j=1}^{t} y^{k+j-1}=\sum_{i=1}^{k-1} h_{i}^{\prime} x^{i}+(t-1) h_{k}^{\prime} x^{k} .
$$

This is an integer relation on $\left\{x^{i}\right\}_{i=1}^{k}$. Therefore, there exists $\alpha \in \mathbb{Z}$ such that

$$
\begin{aligned}
h_{i}^{\prime} & =\alpha h_{i}, \forall i=1, \ldots, k-1, \\
(t-1) h_{k}^{\prime} & =\alpha h_{k}=\alpha .
\end{aligned}
$$

Since $h_{i}>0$ for all $i$ and there is an $i$ such that $h_{i}^{\prime}>0$, equations (4) and (5) imply $\alpha>0$. Therefore, (4) and (5) imply that $h_{i}^{\prime}>0$ for all $i$. Hence, $\bar{h}$ is primitive. 


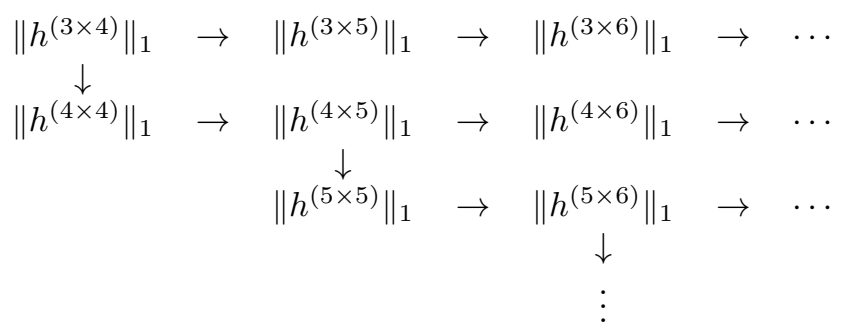

Figure 1: Induction on $t, r$.

Now apply to $\left\{y^{i}\right\}_{i=1}^{k+t-1}$ a permutation of columns so that $y^{k+t-1}$ becomes $\left(v_{1}, u_{r-t+2}, v_{2}, u_{r-t+3}, \ldots, v_{t}, u_{r+1}\right)$. For $i=1, \ldots, k+t-1$, let $\bar{x}^{i}$ be the circuit of $A_{t, r+1}$ which is the image of $y^{i}$ under this permutation. Then $\left\{\bar{x}^{i}\right\}_{i=1}^{k+t-1}$ also satisfy the primitive relation $\sum_{i=1}^{k+t-1} \bar{h}_{i} \bar{x}^{i}=0$ with the same coefficients $\bar{h}$. This completes the proof.

We are now ready to prove Theorem 1.1. In the proof, we use the following notation. Let $\mathscr{A}\left(\left\{x^{i}\right\}_{i=1}^{k}\right)=\left\{\bar{x}^{i}\right\}_{i=1}^{k+t}$ and $\mathscr{B}(h)=\bar{h}=\left(\bar{h}_{1}, \ldots, \bar{h}_{k+t-1}, 1\right)$ denote circuits of $A_{t+1, t+1}$ and the primitive relation which are obtained by the operation of Lemma 3.1 to circuits $\left\{x^{i}\right\}_{i=1}^{k}$ of $A_{t, t+1}$ and the primitive relation $h$. Note that $\|\mathscr{B}(h)\|_{1}=\|h\|_{1}+t$. Furthermore, let $\mathscr{A}^{\prime}\left(\left\{x^{i}\right\}_{i=1}^{k}\right)=$ $\left\{\bar{x}^{i}\right\}_{i=1}^{k+t-1}$ and $\mathscr{B}^{\prime}(h)=\bar{h}=\left(\bar{h}_{1}, \ldots, \bar{h}_{k+t-2}, 1\right)$ denote circuits of $A_{t, r+1}$ and the primitive relation which are obtained by the operation of Lemma 3.2 to circuits $\left\{x^{i}\right\}_{i=1}^{k}$ of $A_{t, r}$ and the primitive relation $h$. Note that $\left\|\mathscr{B}^{\prime}(h)\right\|_{1}=$ $(t-1)\left(\|h\|_{1}-1\right)+t$.

Our proof uses induction on $t, r$. We will construct a primitive relation $h^{(t \times r)}$ on circuits $\mathscr{X}_{(t \times r)}$ of $A_{t, r}$ by induction. Therefore, we obtain $g\left(A_{t, r}\right) \geq$ $\left\|h^{(t \times r)}\right\|_{1}$. Our induction is illustrated in Figure 1. There, a down arrow corresponds to the operation of Lemma 3.1, and a right arrow corresponds to the operation of Lemma 3.2.

Proof of Theorem 1.1. By induction on $t$, we will prove that for all $t \geq 4$ there exist $k(t)=t^{2}-2 t+2$ circuits $\mathscr{X}_{(t \times t)}=\left\{x_{(t \times t)}^{i}\right\}_{i=1}^{k(t)} \subset \mathcal{C}\left(A_{t, t}\right)$ and the primitive relation $h^{(t \times t)}$ such that

$$
\begin{aligned}
x_{(t \times t)}^{k(t)} & =\left(v_{1}, u_{1}, v_{2}, u_{2}, \ldots, v_{t}, u_{t}\right), \\
\sum_{i=1}^{k(t)} h_{i}^{(t \times t)} x^{i} & =0, \\
h_{k(t)}^{(t \times t)} & =1,
\end{aligned}
$$




$$
\left\|h^{(t \times t)}\right\|_{1}=(t-2) !\left(15+\sum_{i=1}^{t-4} \frac{i+4}{(i+2) !}\right) .
$$

Exchange $x^{1}$ and $x^{7}$ of circuits of Example 2.1 and apply to the circuits a permutation of vertices so that

$$
x^{7}=\left(v_{1}, u_{2}, v_{2}, u_{3}, v_{3}, u_{4}\right) .
$$

Let $\mathscr{X}_{(3 \times 4)}=\left\{x_{(3 \times 4)}^{i}\right\}_{i=1}^{7}$ be the image of $\left\{x^{i}\right\}_{i=1}^{7}$ under this permutation. The primitive relation $h^{(3 \times 4)}$ on these circuits satisfy

$$
h^{(3 \times 4)}=(7,2,3,3,5,6,1) .
$$

Notice that $h_{7}^{(3 \times 4)}=1$ holds.

Let $\mathscr{X}_{(4 \times 4)}=\mathscr{A}\left(\mathscr{X}_{(3 \times 4)}\right)$ and $h^{(4 \times 4)}=\mathscr{B}\left(h^{(3 \times 4)}\right)$ denote the image of $\mathscr{X}_{(3 \times 4)}$ and $h^{(3 \times 4)}$ under the operation of Lemma 3.1. Then we have $h^{(4 \times 4)}=(7,2,3,3,5,6,1,1,1,1) \in \mathbb{Z}^{10}$ and

$$
\begin{aligned}
x_{(4 \times 4)}^{10} & =\left(v_{1}, u_{1}, v_{2}, u_{2}, v_{3}, u_{3}, v_{4}, u_{4}\right), \\
h_{10}^{(4 \times 4)} & =1 \\
\left\|h^{(4 \times 4)}\right\|_{1} & =\left\|h^{(3 \times 4)}\right\|_{1}+3=30 .
\end{aligned}
$$

Therefore, we have verified the initial condition at $t=4$ for the induction.

Suppose now that the result holds for $t \geq 4$. Let $\mathscr{X}_{(t \times(t+1))}=\mathscr{A}^{\prime}\left(\mathscr{X}_{(t \times t)}\right)$ and $h^{(t \times(t+1))}=\mathscr{B}^{\prime}\left(h^{(t \times t)}\right)$ denote the image of $\mathscr{X}_{(t \times t)}$ and $h^{(t \times t)} \in \mathbb{Z}^{k(t)}$ under the operation of Lemma 3.2.

$$
\begin{aligned}
x_{(t \times(t+1))}^{k(t)+t-1} & =\left(v_{1}, u_{2}, v_{2}, u_{3}, \ldots, v_{t}, u_{t+1}\right), \\
h_{k(t)+t-1}^{(t \times(t+1))} & =1, \\
\left\|h^{(t \times(t+1))}\right\|_{1} & =(t-1)\left(\left\|h^{(t \times t)}\right\|_{1}-1\right)+t
\end{aligned}
$$

follows from Lemma 3.2. Now let $\mathscr{X}_{((t+1) \times(t+1))}=\mathscr{A}\left(\mathscr{X}_{(t \times(t+1))}\right)$ and $h^{((t+1) \times(t+1))}=\mathscr{B}\left(h^{(t \times(t+1))}\right)$ denote the image of $\mathscr{X}_{(t \times(t+1))}$ and $h^{(t \times(t+1))}$ under the operation of Lemma 3.1. Then

$$
\begin{aligned}
& x_{((t+1) \times(t+1))}^{k(t)+2 t-1}=\left(v_{1}, u_{1}, v_{2}, u_{2}, \ldots, v_{t+1}, u_{t+1}\right), \\
& h_{k(t)+2 t-1}^{((t+1) \times(t+1))}=1,
\end{aligned}
$$




$$
\begin{aligned}
\left\|h^{((t+1) \times(t+1))}\right\|_{1} & =(t-1)\left(\left\|h^{(t \times t)}\right\|_{1}-1\right)+2 t \\
& =(t-1) !\left(15+\sum_{i=1}^{t-4} \frac{i+4}{(i+2) !}\right)+t+1 \\
& =((t+1)-2) !\left(15+\sum_{i=1}^{(t+1)-4} \frac{i+4}{(i+2) !}\right)
\end{aligned}
$$

follows from Lemma 3.1. Here $k(t+1)=k(t)+2 t-1$ and $k(4)=10$ imply $k(t)=t^{2}-2 t+2$. Therefore, the result holds for $t+1$. Henceforth, let $b_{t}=\left\|h^{(t \times t)}\right\|_{1}$.

We fix $t \geq 4$ arbitrarily. We prove by induction on $r$ that, for all $r \geq t$, there are circuits $\mathscr{X}_{(t \times r)}=\left\{x_{(t \times r)}^{i}\right\}_{i=1}^{k(t)} \subset \mathcal{C}\left(A_{t, r}\right)$ and the primitive relation $h^{(t \times r)}$ such that

$$
\begin{aligned}
x_{(t \times r)}^{k(t)} & =\left(v_{1}, u_{r-t+1}, v_{2}, u_{r-t+2}, \ldots, v_{t}, u_{r}\right), \\
\sum_{i=1}^{k(t)} h_{i}^{(t \times r)} x_{(t \times r)}^{i} & =0 \\
h_{k(t)}^{(t \times r)} & =1 \\
\left\|h^{(t \times r)}\right\|_{1} & =(t-1)^{r-t}\left(b_{t}+\frac{1}{t-2}\right)-\frac{1}{t-2} .
\end{aligned}
$$

The initial condition of the induction, at $r=t$, follows from $\left\|h^{(t \times t)}\right\|_{1}=b_{t}$.

Suppose now that the result holds for some $r \geq t$. Let $\mathscr{X}_{(t \times(r+1))}=$ $\mathscr{A}^{\prime}\left(\mathscr{X}_{(t \times r)}\right)$ and $h^{(t \times(r+1))}=\mathscr{B}^{\prime}\left(h^{(t \times r)}\right)$ denote the image of $\mathscr{X}_{(t \times r)}$ and $h^{(t \times r)}$ under the operation of Lemma 3.2. Then

$$
\begin{aligned}
x_{(t \times(r+1))}^{k(t)+t-1} & =\left(v_{1}, u_{r-t+2}, v_{2}, u_{r-t+3}, \ldots, v_{t}, u_{r+1}\right), \\
h_{k(t)+t-1}^{(t \times(r+1))} & =1, \\
\left\|h^{(t \times(r+1))}\right\|_{1} & =(t-1)\left(\left\|h^{(t \times r)}\right\|_{1}-1\right)+t \\
& =(t-1)\left((t-1)^{r-t}\left(b_{t}+\frac{1}{t-2}\right)-\frac{1}{t-2}-1\right)+t \\
& =(t-1)^{r+1-t}\left(b_{t}+\frac{1}{t-2}\right)-\frac{1}{t-2}
\end{aligned}
$$


Table 4: Circuits of $A_{4,4}$

\begin{tabular}{c|cccc|c} 
& $u_{1}$ & $u_{2}$ & $u_{3}$ & $u_{4}$ & \\
\hline $\bar{x}^{7}=\left(v_{1}, u_{2}, v_{4}, u_{1}, v_{3}, u_{4}\right)=$ & 0 & 1 & 0 & -1 & $v_{1}$ \\
& 0 & 0 & 0 & 0 & $v_{2}$ \\
\hline $\bar{x}^{8}=\left(v_{2}, u_{3}, v_{4}, u_{2}\right)=$ & -1 & 0 & 0 & 1 & $v_{3}$ \\
& 1 & -1 & 0 & 0 & $v_{4}$ \\
\hline $\bar{x}^{9}=\left(v_{3}, u_{4}, v_{4}, u_{3}\right)=$ & 0 & 0 & 0 & 0 & $v_{1}$ \\
& 0 & -1 & 1 & 0 & $v_{2}$ \\
$\bar{x}^{10}=\left(v_{1}, u_{2}, v_{2}, u_{3}, v_{3}, u_{1}, v_{4}, u_{4}\right)=$ & 0 & 0 & 0 & 0 & $v_{3}$ \\
& 0 & 0 & 0 & 0 & $v_{1}$ \\
\hline & 0 & 0 & 0 & 0 & $v_{2}$ \\
& 0 & 0 & -1 & 1 & $v_{3}$ \\
& 0 & 0 & 1 & -1 & $v_{4}$ \\
\hline
\end{tabular}

follows from Lemma 3.2. Therefore, the result holds for $r+1$ and

$$
g\left(A_{t, r}\right) \geq(t-1)^{r-t}\left(b_{t}+\frac{1}{t-2}\right)-\frac{1}{t-2}
$$

follows from Lemma 2.2.

\section{Discussion}

In this paper, we provided a lower bound in Theorem 1.1 by the induction on $t, r$. Here we discuss some ideas for improving our lower bound.

Look at Figure 1 again. On the step $\left\|h^{(3 \times 4)}\right\|_{1} \rightarrow\left\|h^{(4 \times 4)}\right\|_{1}$, we can construct a larger primitive relation than the relation constructed in the proof.

Example 4.1. Let $\left\{x^{i}\right\}_{i=1}^{7}$ denote the circuits in Example 2.1. Using the natural embedding of $K_{3,4}$ into $K_{4,4}$, let

$$
\bar{x}^{i}=x^{i}, \forall i=1, \ldots, 6,
$$

and for $i=7, \ldots, 10$, we define $\bar{x}^{i}$ as shown in Table 4 . Then $\left\{\bar{x}^{i}\right\}_{i=1}^{10}$ are circuits of $\operatorname{ker}_{\mathbb{Z}}\left(\mathbf{1}_{4}^{(4)}\right)$ and $\bar{h}=(2,4,6,6,10,12,7,7,7,7)$ is its primitive relation. 
Then, by Proposition 2.2,

$$
g\left(\mathbf{1}_{4}^{(4)}\right) \geq 2+4+6+6+10+12+7+7+7+7=68 .
$$

Equation (6) is sharper than the evaluation in Theorem 1.1.

We could start induction from circuits and its primitive relation in Example 4.1. Then we obtain a sharper evaluation for some small $t, r$. However, it unfortunately turns out that, if we start from Example 4.1 then on the step $\left\|h^{(8 \times r)}\right\|_{1} \rightarrow\left\|h^{(8 \times(r+1))}\right\|_{1}$, we cannot obtain an exponential lower bound. Therefore, we did not use Example 4.1 in the proof of Theorem 1.1. However, this example suggests that there may be some other better initial set of circuits for our induction.

\section{References}

[1] S. Aoki and A. Takemura (2003). Minimal basis for a connected Markov chain over $3 \times 3 \times K$ contingency tables with fixed two-dimensional marginals. Aust. N. Z. J. Stat. 45 229-249. MR1983834

[2] Y. Berstein and S. Onn (2009). The Graver complexity of integer programming. Ann. Comb. 13 289-296. MR2557039

[3] J. A. De Loera and S. Onn (2006). All linear and integer programs are slim 3-way transportation programs. SIAM J. Optim. 17 806-821. MR2257210

[4] J. A. De Loera, R. Hemmecke, S. Onn, and R. Weismantel (2008). $n$-fold integer programming. Discrete Optim. 5 231-241. MR2408419

[5] S. Hoşten and S. Sullivant (2007). A finiteness theorem for Markov bases of hierarchical models. J. Combin. Theory Ser. A 114 311-321. MR2293094

[6] S. Onn (2010). Nonlinear Discrete Optimization. An Algorithmic Theory. Zurich Lectures in Advanced Mathematics. European Mathematical Society (EMS), Zürich. MR2724387

[7] F. Santos and B. Sturmfels (2003). Higher Lawrence configurations. J. Combin. Theory Ser. A 103 151-164. MR1986836

[8] B. Sturmfels (1996). Gröbner Bases and Convex Polytopes. American Mathematical Society, Providence, Rhode Island. MR1363949 
TAisei Kudo

Graduate School of Information Science and Technology The UNIVERSITY OF TOKYO

Hongo 7-3-1, Bunkyo-Ku, TOKYO 113-8656

JAPAN

E-mail address: n6d2ji5k@gmail.com

AKIMIChi TAKEMURA

Graduate School of Information Science and Technology THE UNIVERSITY OF TOKYO

HONGO 7-3-1, BUnKYO-KU, TOKYO 113-8656

JAPAN

E-mail address: takemura@stat.t.u-tokyo.ac.jp

ReCeived FeBruary 23, 2011 